\title{
Clinical significance of LSECtin and its association with PVR in non-small-cell lung cancer patients
}

\author{
Yizhong Zhang ${ }^{1 \#}$, Huanling Lai ${ }^{1 \#}$, Peipei Chen ${ }^{2 \#}$, Dan $\mathrm{Li}^{3}$, Imran Khan ${ }^{1}$, Wen Luan Wendy Hsiao ${ }^{1}$, \\ Xingxing Fan ${ }^{1}$, Xiaojun Yao ${ }^{1}$, Qibiao Wu ${ }^{1}$, Meifang Wang ${ }^{2,4}$, Elaine Laihan Leung ${ }^{1}$
}

${ }^{1}$ State Key Laboratory of Quality Research in Chinese Medicine, Macau Institute For Applied Research in Medicine and Health, Macau University of Science and Technology, Macau (SAR), China; ${ }^{2}$ Department of Respiratory and Critical Care Medicine, Taihe Hospital, Hubei University of Medicine, Shiyan, China; ${ }^{3}$ Department of Pathology, Taihe Hospital, Hubei University of Medicine, Shiyan, China; ${ }^{4}$ Hubei Key Laboratory of Embryonic Stem Cell Research, Hubei University of Medicine, Shiyan, China

Contributions: (I) Conception and design: EL Leung, M Wang, Q Wu; (II) Administrative support: EL Leung, M Wang, Q Wu; (III) Provision of study materials or patients: EL Leung, M Wang, Q Wu; (IV) Collection and assembly of data: Y Zhang, H Lai, P Chen, D Li; (V) Data analysis and interpretation: Y Zhang, H Lai, I Khan, WLW Hsiao, X Fan, X Yao; (VI) Manuscript writing: All authors; (VII) Final approval of manuscript: All authors.

\#These authors contributed equally to this work.

Correspondence to: Elaine Laihan Leung. State Key Laboratory of Quality Research in Chinese Medicine, Macau Institute for Applied Research in Medicine and Health, Macau University of Science and Technology, Macau (SAR), China. Email: lhleung@must.edu.mo. Meifang Wang. Department of Respiratory and Critical Care Medicine, Taihe Hospital, Hubei University of Medicine, Shiyan, China. Email: wmfpps02@hotmail.com. Qibiao Wu. State Key Laboratory of Quality Research in Chinese Medicine, Macau Institute for Applied Research in Medicine and Health, Macau University of Science and Technology, Macau (SAR), China. Email: qbwu@must.edu.mo.

Background: Liver and lymph node sinusoidal endothelial cell C-type lectin (LSECtin) is one of the new generation immune checkpoint ligand molecules and plays an important role in the immune environment. Poliovirus receptor (PVR), as another immunosuppression-related molecule, is upregulated in various malignant tumors. However, the clinical value of LSECtin and the correlation of LSECtin with PVR in nonsmall-cell lung cancer (NSCLC) remain to be elucidated. In this study, a retrospective study was performed to address these issues.

Methods: This retrospective study included 98 patients with NSCLC. Immunohistochemistry (IHC) was used to detect the expression of LSECtin and PVR in the paraffin-embedded tumor tissue specimens. LSECtin was analyzed for associations with the survival rate and overall survival (OS) of the subjects. The mRNA expression of LSECtin and PVR was assessed using the expression data from The Cancer Genome Atlas (TCGA) database. Clinical characteristics, prognosis, and the expression of LSECtin and PVR were included in the statistical analysis.

Results: High positive rates of LSECtin were found in the patients with NSCLC who were nonsmokers, at advanced stages, or had lung adenocarcinoma. Patients with positive LSECtin expression had a significantly lower survival rate $(\mathrm{P}=0.008)$ and shorter OS $(\mathrm{P}=0.017)$ than those with negative LSECtin. Significant correlation was found between the LSECtin and PVR expression in the patients with NSCLC $(\mathrm{P}<0.001)$.

Conclusions: The increased expression of LSECtin was related to the poor prognosis of patients with NSCLC after tumor resection and has the potential value for predicting the prognosis of these patients. The positive correlation between LSECtin and PVR in NSCLC provides a theoretical basis for the future combination therapy of immune checkpoints.

Keywords: Liver and lymph node sinusoidal endothelial cell C-type lectin (LSECtin); poliovirus receptor; nonsmall cell lung cancer (NSCLC); immune checkpoint; overall survival

Submitted May 01, 2020. Accepted for publication Sep 01, 2020.

(c) Annals of Translational Medicine. All rights reserved. 
doi: 10.21037/atm-20-3665

View this article at: http://dx.doi.org/10.21037/atm-20-3665

\section{Introduction}

Lung cancer is one of the cancer types with the highest morbidity and mortality worldwide. As the most common histologic subtype of lung cancer, non-small cell lung cancer (NSCLC) has various treatments and strategies (1), but the 5-year survival rate for patients with NSCLC is not favorable. In cancer therapy, immune checkpoint inhibitors are used to block or activate the relevant pathways to suppress tumor immune escape or tumor progression. Extensive studies have been performed on the functions of classic immune checkpoint regulating the pro-inflammatory $\mathrm{T}$ cell response and maintaining self-tolerance (2). The successful blockade of cytotoxic $\mathrm{T}$ lymphocyte-associated antigen 4 (CTLA-4) or programmed death 1 (PD-1)/ programmed death ligand 1 (PD-L1) pathways in clinical trials has greatly enhanced the strategies for NSCLC treatment in long-term drug resistance environment (3). Despite the encouraging results from immune checkpoint therapy, recent studies have shown that the overall efficiency of anti-PD-1/PD-L1 immunotherapy in patients with NSCLC remains unsatisfactory. The response rate is only approximately $20 \%$, and most patients show acquired resistance even during the early treatment stage (4). These records indicate that new inhibitory pathways beyond CTLA-4 and PD-1/PD-L1 are urgently needed to treat patients with NSCLC. Lymphocyte-activation gene 3 (LAG-3), T-cell immunoglobulin and mucin-domain containing-3 (TIM-3), and T cell immunoglobulin and ITIM domain (TIGIT) pathways have attracted the most attention in the hot topic of next-generation immune checkpoint (5).

LAG-3, TIM-3, and TIGIT are receptors expressed in the plasma membrane of immune cells. Similar to PD-1, these receptors have corresponding ligands expressed in the tumor cell membrane. The ligand on the tumor cell surface that binds to the receptor of the immune cell surface will also promote tumor cell escape from immune surveillance (5). Blocking these pathways of immune checkpoint inhibitors (ICIs) could enhance antitumor immune responses. Coexpression of LAG-3 and PD-1 is found to be upregulated on several tumor-infiltrating lymphocytes (TILs), resulting in immune exhaustion and promotion of tumor cell proliferation (6-8). This reaction could also improve other kinds of immunotherapy because of its various action mechanisms primarily by preventing the cell cycle process (9). Similar to PD-1 and PD-L1 pair, blocking the ligands of the ICIs on the tumor cell surface is also crucial to reduce tumor immune suppression. These ligands could be important biomarkers in clinical diagnosis, treatment selection, and prognosis.

As one of the ligands of the immune checkpoint molecule LAG-3, the liver and lymph node sinusoidal endothelial cell C-type lectin (LSECtin) mediated the mechanism of tumor immune escape in melanoma (10). LSECtin was first discovered to be expressed in sinusoidal endothelial cells of the liver and lymph nodes (11). LSECtin belongs to the calcium-dependent lectin family (11). Studies had confirmed that LSECtin not only acted as an adhesion factor for exogenous pathogens $(12,13)$ but also played an important role in autoimmune regulation (14). Physiologically, LSECtin inhibited activated T cells in the liver and protected the liver from excessive immune responses (14). LSECtin could also suppress CD4(+) T cell proliferation and interleukin-2 (IL-2) production by upregulating the expression of casitas B-lineage lymphoma B (Cbl-b) (15). Moreover, LSECtin-Butyrophilin Subfamily 3 Member A3 (BTN3A3) axis was involved in the mediation of the differentiation of macrophages and had an important role in enhancing the stemness of breast cancer in the tumor environment (16). However, the role of LSECtin in the progression of NSCLC remains unclear.

The poliovirus receptor (PVR) is another molecule related to the immunosuppression caused by tumor cells. In the authors' previous studies, PVR, the ligand of TIGIT, was positively expressed in most patients with NSCLC and significantly related to the overall survival (OS) (17). Moreover, PVR expression was positively correlated with CTLA-4 in clinical surgically resected NSCLC, exploring the important role of PVR in immune response (17). TILs often highly express high levels of TIGIT, consistent with a dysfunctional phenotype $(18,19)$. The expression of LAG-3 and TIGIT on TILs was significantly related to PD-1 in cutaneous melanoma (20). Anti-LAG-3 monoclonal antibody (BMS-986016) is one of the antiLAG-3 antibodies under investigation, and the clinical 
trials of BMS-986016 alone or the combination of BMS986016 and Nivolumab for several malignant tumors are still in the clinical evaluation stage (21). Crosstalk between the regulatory pathways of the ICI ligands on the tumor cells could also be a key immune-modulating mechanism. Understanding the relationship between different ligands on tumors can provide additional information for the clinical application of their inhibitors as a treatment for cancer. In this study, the expression and prognostic significance of LSECtin in patients with NSCLC were investigated. At the same time, the correlation between the expression of LSECtin and PVR was explored in the NSCLC samples. The following article is presented in accordance with the REMARK reporting checklist (available at http://dx.doi. org/10.21037/atm-20-3665).

\section{Methods}

\section{Patients}

Tumor tissue samples were collected from 98 patients with NSCLC who successfully underwent tumor resection in Hubei Taihe Hospital from 2014 to 2017. The samples were paraffin-embedded tumor sections and stored at $4{ }^{\circ} \mathrm{C}$. Relevant patient statistics, including the time of diagnosis, gender, age, smoking history, pathological pattern, degree of tumor differentiation, and tumor stage, were collected using an electronic medical record system. According to the pathological examination, all NSCLC cases were diagnosed into two types, namely, 50 cases of lung adenocarcinoma (LUAD) and 48 cases of lung squamous cell carcinoma (LUSC). The tumor stage was determined according to the American Joint Committee on Cancer and Union for International Cancer Control. The standard for age classification was 65 years old. Meanwhile, long-term follow-up of all patients was conducted through clinical observation or telephone communication. The median follow-up time for all cases was 56.95 months. The followup deadline for all cases was January 2020. The study was conducted in accordance with the Declaration of Helsinki (as revised in 2013) and had been approved by the Institutional Research Ethics Committee of Hubei Taihe Hospital (2020KS014). All patients signed informed consent.

\section{Immunobistochemistry}

Paraffin-embedded sections ( $4 \mu \mathrm{m}$ thick) were dewaxed in xylene and hydrated with a graded ethanol solution.
All samples were stained with an immunohistochemistry kit (K8002; Dako, Glostrup, Denmark). The primary antibodies used were anti-LSECtin (1:200 dilution; Abcam) and anti-PVR (1:200 dilution; Cell Signaling). Each section was counterstained with hematoxylin.

\section{Immunobistochemical (IHC) scoring}

The IHC staining results were blindly evaluated by two independent pathologists using an optical microscope. According to the percentage of positively stained cells and staining intensity, the IHC score was determined by a semiquantitative method. The percentages of positively stained cells were scored as $0(<5 \%), 1+(6-25 \%), 2+(25-50 \%)$, $3+(50-75 \%)$, and $4+(>75 \%)$. The staining intensity was categorized as follows: 0 , no staining; 1 , weak staining; 2 , moderate staining; and 3 , strong staining. Two results were multiplied to obtain an IHC score. Finally, high expression (score $=2$ or 3 ) and low expression $($ score $>3$ ) were defined as positive, and the expression was negative when the score was 0 .

\section{Statistical analysis}

All statistical analyses were performed using the IBM SPSS statistical software version 25.0 . The $\chi^{2}$-test was used to assess the relationship between categorical variables and the correlation between LSECtin and PVR expression levels. The capability of LSECtin expression to identify pathological types in NSCLC was evaluated by the receiver operating characteristic (ROC) curves. The effect of clinical variables on the LSECtin expression was determined by logistic regression analysis. The OS of patients was defined as the time from diagnosis to death or last follow-up time. Survival results were analyzed by Kaplan-Meier curves and log-rank test. Univariate and multivariate analyses of the survival data were performed by using Cox models. All statistical tests were two-sided, and $\mathrm{P}<0.05$ was considered as statistically significant.

\section{TCGA database analysis}

The data of mRNA expression in several types of tumor were collected from TCGA databases. These tumors included LUAD ( $\mathrm{n}=576)$, LUSC $(\mathrm{n}=553)$, liver cancer $(n=423)$, and colon cancer $(n=329)$. The data were normalized in $\log 2$ (norm_count 1 ). Then, RStudio (1.2.5033) library ggpubr (0.0.2) was used to analyze and 

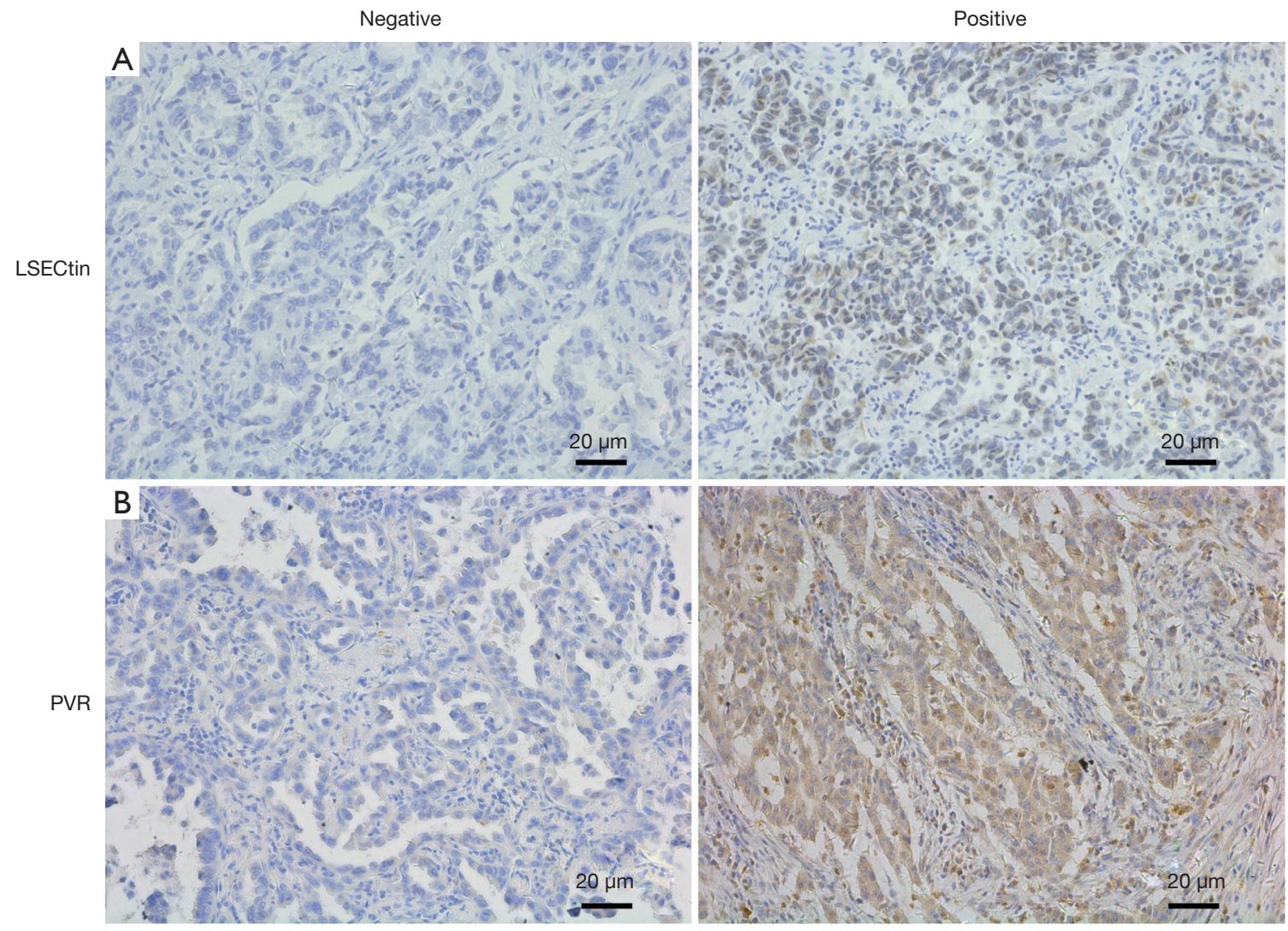

Figure 1 LSECtin and PVR expression in NSCLC tumor tissues. (A) Expression of LSECtin in NSCLC. (B) Expression of PVR in NSCLC. Original magnification, $\times 200$. LSECtin, liver and lymph node sinusoidal endothelial cell C-type lectin; PVR, poliovirus receptor; NSCLC, non-small cell lung cancer.

plot all data with Pearson correlation method. In addition, $\mathrm{P}<0.05$ was considered as statistically significant.

\section{Results}

\section{Patient characteristics}

A total of 98 patients with NSCLC, including 78 males (79.6\%) and 20 females $(20.4 \%)$, were included in this study. The average age of patients at the time of diagnosis was 58 years (range, 35-77 years). Clinicopathological diagnosis showed that 50 patients had LUAD and 48 patients had LUSC. In terms of the tumor node metastasis (TNM) stage, 49 (50.0\%), 30 (30.6\%), 17 (17.4\%), and $2(2.0 \%)$ patients were determined to be in stages I, II, III, and IV, respectively. A total of 68 cases had moderate differentiation, accounting for the largest proportion of all cases. The numbers of poorly and highly differentiated cases were 22 and 8 cases, respectively. In addition, the smoking history, which was a peculiar factor affecting lung disease, was investigated. The results showed that $68.4 \%$ of patients had a smoking history $(\mathrm{n}=67)$. Finally, 25 patients died, and 4 patients lost contact.

\section{Association of LSECtin expression with the clinicopathological characteristics}

The immunohistochemistry images showed that the positive staining of LSECtin was mainly located in the cytoplasm of cells, with a small amount of nuclear staining (Figure 1). First, the association of LSECtin expression was evaluated with the clinicopathological information of the patients with NSCLC, and the results are summarized in Table 1. The positive rate of LSECtin in patients with LUAD was significantly higher than that in LUSC $(\mathrm{P}<0.001)$. The data showed that the degree of tumor progression was also related to the expression of LSECtin, 
Table 1 Association of the expression of LSECtin and clinicopathological characteristics in NSCLC patients

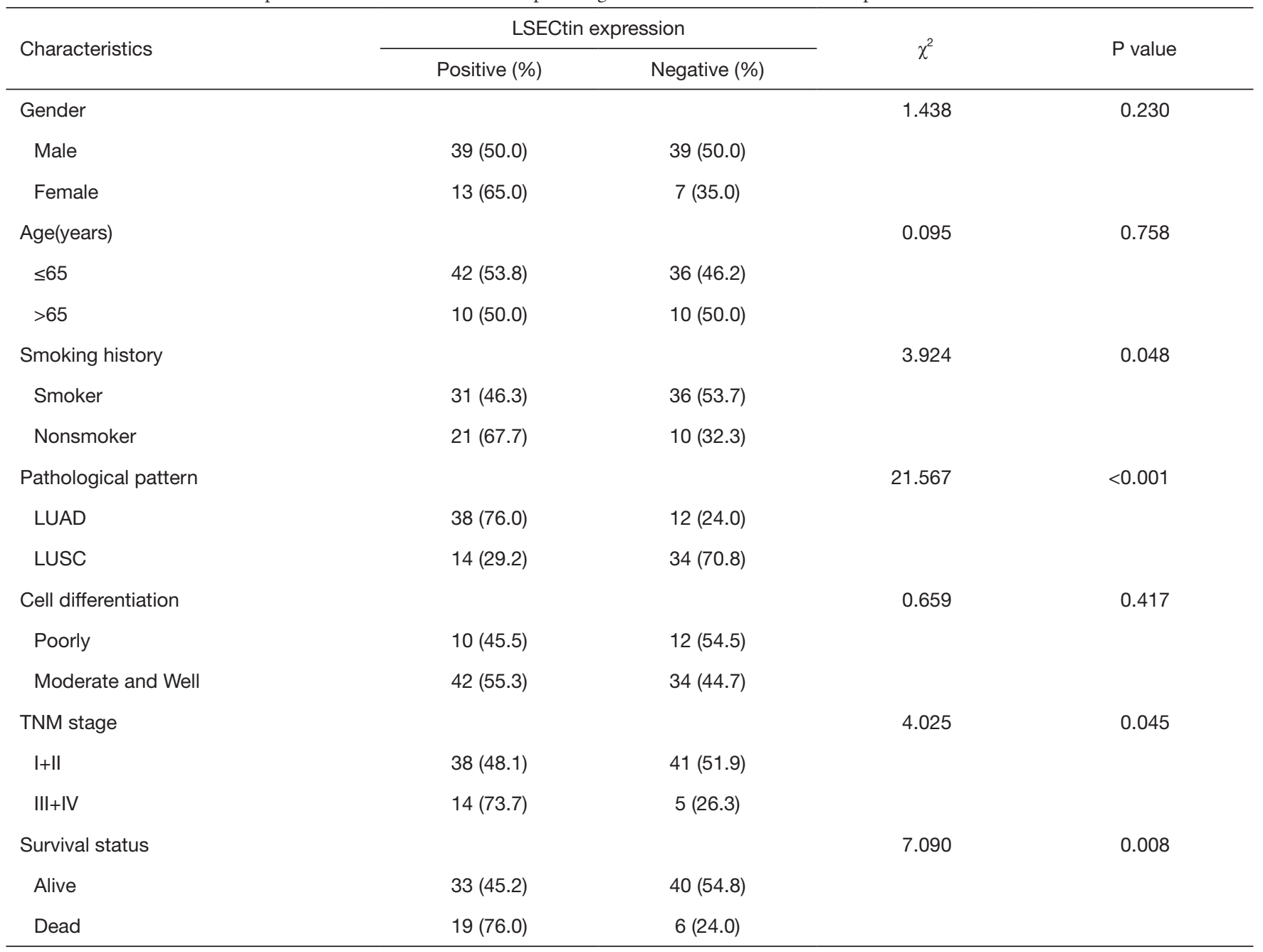

LSECtin, liver and lymph node sinusoidal endothelial cell C-type lectin; NSCLC, non-small cell lung cancer; LUAD, lung adenocarcinoma; LUSC, lung squamous cell carcinoma; TNM, tumor node metastasis.

and the LSECtin positive rate of patients with advanced NSCLC was significantly increased $(\mathrm{P}=0.045)$. The positive rate of LSECtin in nonsmokers was interestingly higher than in smokers $(\mathrm{P}=0.048)$. No significant statistical difference was found between LSECtin expression and other clinical information (including gender, age, and tumor differentiation). Then, a logistic regression analysis of clinical factors was performed (Table 2). Univariate results showed that smoking history $(\mathrm{P}=0.050)$ and pathological pattern $(\mathrm{P}<0.001)$ were related to tumor LSECtin expression. In multiple regression analysis, pathological classification $(\mathrm{P}<0.001)$ and tumor stage $(\mathrm{P}=0.010)$ were independently correlated with tumor LSECtin expression.

\section{Significance of pathological pattern and smoking history on LSECtin expression in different conditions}

The pathological pattern is an important indicator of the early diagnosis of cancer and instructive for subsequent cancer treatment. Given its specificity, smoking history has become one of the factors used as foci in clinical lungrelated diseases. The results show that the expression of LSECtin was associated with pathological patterns and smoking history in patients with NSCLC (Tables 1,2). To further explore the interaction between the clinical factors, a multilayer $\chi^{2}$-test was performed to compare the effects of various factors on the LSECtin expression with different pathological types (Table 3) and smoking habits (Table 4). 
Table 2 Univariate and multivariate analysis of clinicopathological characteristics correlated with LSECtin expression in NSCLC patients

\begin{tabular}{|c|c|c|c|c|}
\hline Characteristics & \multicolumn{2}{|c|}{ Univariate analysis } & \multicolumn{2}{|c|}{ Multivariate analysis } \\
\hline \multicolumn{5}{|l|}{ Age (years) } \\
\hline$\leq 65$ & 1 & & & \\
\hline$>65$ & $1.167(0.437-3.118)$ & 0.759 & & \\
\hline \multicolumn{5}{|l|}{ Gender } \\
\hline Male & 1 & & & \\
\hline Female & $0.538(0.194-1.494)$ & 0.234 & & \\
\hline \multicolumn{5}{|l|}{ Smoking history } \\
\hline Smoker & 1 & & & \\
\hline LUAD & 1 & & 1 & \\
\hline LUSC & 7.690 (3.129-18.901) & $<0.001$ & $12.573(3.747-42.187)$ & $<0.001$ \\
\hline \multicolumn{5}{|l|}{ Cell differentiation } \\
\hline Poor & 1 & & & \\
\hline Moderate and Well & $0.675(0.260-1.750)$ & 0.418 & & \\
\hline \multicolumn{5}{|l|}{ TNM stage } \\
\hline $1+I I$ & 1 & & 1 & \\
\hline III+IV & $0.331(0.109-1.007)$ & 0.051 & $0.173(0.046-0.655)$ & 0.010 \\
\hline
\end{tabular}

LSECtin, liver and lymph node sinusoidal endothelial cell C-type lectin; NSCLC, non-small cell lung cancer; LUAD, lung adenocarcinoma; LUSC, lung squamous cell carcinoma; TNM, tumor node metastasis; OR, odds ratio; CI, confidence interval.

The effects of TNM stage on the expression of LSECtin in the samples were significantly different in the two NSCLC pathological types. LSECtin expression was not affected by the TNM stage in patients with LUAD. However, in patients with LUSC, the positive rate in early-stage patients was lower than that in advanced patients $(\mathrm{P}=0.016)$. The LSECtin positive rate of nonsmokers was previously found to be higher than that of smokers, and this phenomenon should be mainly affected by the pathological type of the patients with NSCLC. Most patients with smoking history were diagnosed with LUSC $(\mathrm{P}<0.001)$, and the positive expression of LSECtin in patients with LUAD was dominant. The capability of LSECtin was assessed from the ROC curve to identify the pathological typing of NSCLC (Figure 2). The results showed that the area under the curve was $0.756(\mathrm{P}<0.001)$, and the LSECtin expression could be used to distinguish the pathological types with $70.8 \%$ specificity and $76.0 \%$ sensitivity.

\section{Relationship between the expression of LSECtin and PVR}

Blocking of classical immune checkpoint PD-L1 ligands on multiple cancer types had produced promising clinical results. LSECtin and PVR were the ligands of LAG-3 and TIGIT, respectively. To investigate the expression of the two molecules in NSCLC, the PVR expression was simultaneously detected in this batch of tumor tissues by IHC staining. PVR was found to be mainly expressed in the tumor cells, and a small amount of positive staining was distributed in the lymphatic infiltrating cells (Figure 1). Then, $\chi^{2}$ analysis was used to describe the association between the expression of LSECtin and PVR in patients with NSCLC (Table 5). Interestingly, the expression of LSECtin was positively correlated with PVR in patients with NSCLC $(\mathrm{P}<0.001)$.

The mRNA expression of LSECtin and PVR was analyzed in several cancers by using the TCGA database 
Table 3 Multilayer statistical analysis of association among pathological pattern, LSECtin expression and clinicopathological characteristics in NSCLC patients

\begin{tabular}{|c|c|c|c|c|}
\hline \multirow{2}{*}{ Clinicopathological Characteristics } & \multicolumn{4}{|c|}{ Pathological pattern } \\
\hline & LSECtin positive & LSECtin negative & LSECtin positive & LSECtin negative \\
\hline \multicolumn{5}{|l|}{ Gender } \\
\hline Male & 25 & 6 & 14 & 33 \\
\hline$P$ value & \multicolumn{2}{|c|}{0.326} & \multicolumn{2}{|c|}{0.517} \\
\hline \multicolumn{5}{|l|}{ Age } \\
\hline$\leq 65$ & 32 & 10 & 10 & 26 \\
\hline$>65$ & 6 & 2 & 4 & 8 \\
\hline Smoker & 17 & 5 & 14 & 31 \\
\hline Non-smoker & 21 & 7 & 0 & 3 \\
\hline$P$ value & \multicolumn{2}{|c|}{0.852} & \multicolumn{2}{|c|}{0.251} \\
\hline \multicolumn{5}{|l|}{ Cell differentiation } \\
\hline Poor & 6 & 2 & 4 & 10 \\
\hline Moderate and Well & 32 & 10 & 10 & 24 \\
\hline$P$ value & \multicolumn{2}{|c|}{0.942} & \multicolumn{2}{|c|}{0.954} \\
\hline \multicolumn{5}{|l|}{ TNM stage } \\
\hline
\end{tabular}

LSECtin, liver and lymph node sinusoidal endothelial cell C-type lectin; NSCLC, non-small cell lung cancer; LUAD, lung adenocarcinoma; LUSC, lung squamous cell carcinoma; TNM, tumor node metastasis.

(Figures S1-S4). In the correlation analysis, the expression of LSECtin and PVR mRNA was found to have a trend of positive correlation in LUSC $(\mathrm{P}=0.066)$. The correlation analysis of LSECtin and PVR in the samples from patients with NSCLC with different pathological patterns is shown in Table S1. The expression of LSECtin and PVR was positively correlated in patients with LUSC, which was consistent with the results from the TCGA database. Although the correlation between these two molecules in LUAD had no significant statistical correlation, the data showed a certain positive correlation. Meanwhile, the probability of double-positive LSECtin and PVR in patients with LUAD was greater than that in patients with LUSC.
These results still need to be verified by expanding the sample size in subsequent studies.

\section{Association among LSECtin expression and survival outcome with NSCLC}

The patients were followed up regularly, and 25 patients had died by January 2020 . The $\chi^{2}$ results showed that, in all 98 NSCLC cases, the survival rate of patients with positive LSECtin expression was significantly lower than those with negative LSECtin ( $\mathrm{P}=0.008$, Table 1). Meanwhile, Figure 3 also shows that the survival time of the LSECtin-positive group was significantly shorter than that in the negative 
Table 4 Multilayer statistical analysis of association among smoking history, LSECtin expression and clinicopathological characteristics in NSCLC patients

\begin{tabular}{|c|c|c|c|c|}
\hline \multirow{2}{*}{ Clinicopathological characteristics } & \multicolumn{4}{|c|}{ Smoking history } \\
\hline & LSECtin positive & LSECtin negative & LSECtin positive & LSECtin negative \\
\hline \multicolumn{5}{|l|}{ Gender } \\
\hline Male & 31 & 36 & 8 & 3 \\
\hline$P$ value & \multicolumn{2}{|c|}{ / } & \multicolumn{2}{|c|}{0.660} \\
\hline \multicolumn{5}{|l|}{ Age } \\
\hline$\leq 65$ & 24 & 29 & 18 & 7 \\
\hline$>65$ & 7 & 7 & 3 & 3 \\
\hline LUAD & 17 & 5 & 21 & 7 \\
\hline LUSC & 14 & 31 & 0 & 3 \\
\hline$P$ value & \multicolumn{2}{|c|}{$<0.001$} & \multicolumn{2}{|c|}{0.008} \\
\hline \multicolumn{5}{|l|}{ Cell differentiation } \\
\hline Poor & 9 & 11 & 1 & 1 \\
\hline Moderate and Well & 22 & 25 & 20 & 9 \\
\hline$P$ value & \multicolumn{2}{|c|}{0.892} & \multicolumn{2}{|c|}{0.579} \\
\hline \multicolumn{5}{|l|}{ TNM stage } \\
\hline
\end{tabular}

LSECtin, liver and lymph node sinusoidal endothelial cell C-type lectin; NSCLC, non-small cell lung cancer; LUAD, lung adenocarcinoma; LUSC, lung squamous cell carcinoma; TNM, tumor node metastasis.

group $(\mathrm{P}=0.017)$. The mean survival time of patients with NSCLC was 65.67 months in the negative group and was only 50.92 months in the positive group. Given that the effects of pathological typing and smoking history on LSECtin expression had been confirmed, pathological typing and smoking history were also included in the OS analysis (Figure 4). The OS of patients in the adenocarcinoma group $(\mathrm{P}=0.033)$ and the nonsmoker group $(\mathrm{P}=0.011)$ were evidently susceptible to LSECtin positive expression. As shown in Table 6, univariate analysis indicated that the TNM stage and LSECtin expression were key indicators related to OS. Moreover, multivariate analysis showed that pathological typing $(\mathrm{P}=0.030)$ and tumor stage $(\mathrm{P}<0.001)$ could be identified as independent predictors of OS.

\section{Discussion}

In this study, the expression of LSECtin in the tumor tissues of 98 patients with NSCLC was evaluated. The results showed that LSECtin could be detected in the tumor cells of the patients, and LSECtin positive expression was related to several clinical factors, including smoking history, pathological typing, and TNM stage. In addition, the patients with positive expression of LSECtin had a significantly lower OS rate than the negative group. Furthermore, the results indicated a positive correlation 
between the expression of LSECtin and PVR in the tumor cells of patients with NSCLC. These data suggested that LSECtin could be a clinical immunotherapy biomarker for NSCLC.

Previous studies have reported that CD44 was an "endogenous" ligand of LSECtin (22), while the CD44 family was involved in the regulation of the extracellular matrix and cell migration $(23,24)$. LSECtin has been associated with tumor migration. In colon cancer, the liver metastasis of LS174T and LoVo cells in LSECtin-knockout nude mice was significantly reduced compared with the control group (25). In addition, the study also showed that the serum levels of soluble LSECtin in patients with metastatic colon cancer was higher than in nonmetastatic colon cancer (25). Another type C lectin DC-SIGNR,

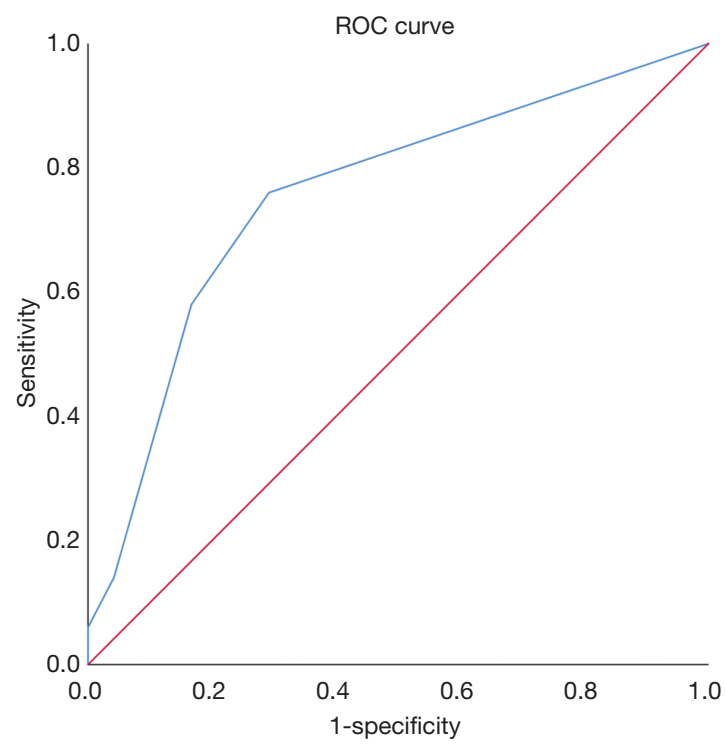

Figure 2 ROC curve of LSECtin expression to discriminate pathological pattern in NSCLC. LSECtin, liver and lymph node sinusoidal endothelial cell C-type lectin; NSCLC, non-small cell lung cancer; ROC, receiver operating characteristic. which was also homologous to LSECtin, had also been proved to present the same role in cancer (26). Local recurrence and distant metastasis had important impact on the prognosis of Chinese patients with NSCLC. In the data statistics of all NSCLC tumor samples, the positive rate of LSECtin increased in LUAD and advanced patients, and LSECtin positive expression was associated with poor prognosis. Patients with adenocarcinoma had a high risk of metastasis (27), and most advanced cancer patients may have long-distant-organ metastasis when they were diagnosed. Therefore, LSECtin could be used as a potential biomarker to predict the prognosis of NSCLC.

The important role of LSECtin in the immune system has been highlighted in recent studies. LSECtin mediated the release of anti-inflammatory factors from macrophages to direct apoptotic cell clearance and intestinal repair in experimental colitis (28). In a melanoma study, the combination of LSECtin and LAG-3 suppressed tumorspecific $\mathrm{T}$ cell immune responses (10). In the present study, analysis of the IHC results showed that the expression of LSECtin and PVR was positively correlated in the tumor tissues. PVR and LSECtin belonged to the immune co-inhibitory ligands in tumor cells, and the immunosuppressive receptors of PVR included TIGIT and CD96 (5). PVR had been reported to be highly expressed in various malignancies (29). PVR could cause immune suppression by regulating the activity of $\mathrm{T}$ cells and natural killer (NK) cells (29,30). Myeloid-derived cells had a wide range of immunosuppressive effects, and PVR and LSECtin could be found in myeloid-derived cells. LSECtin existed in various myeloid-derived cells, which are related to antigen capture and recognition in the human body (31). The expression of PVR in the melanoma cells and tumorinfiltrating myeloid cells influenced the efficacy of antiPD-1 or and anti-CTLA4 immunotherapy (32). Moreover, the LAG-3 and TIGIT pathways involved in LSECtin and PVR had been analyzed in some related immunotherapy

Table 5 Association of LSECtin and PVR expression in NSCLC patients

\begin{tabular}{|c|c|c|c|c|}
\hline PVR & \multicolumn{2}{|c|}{ LSECtin } & $\chi^{2}$ & $\mathrm{P}$ \\
\hline Positive & 38 & 14 & 17.820 & $<0.001$ \\
\hline Negative & 14 & 32 & & \\
\hline
\end{tabular}

LSECtin, liver and lymph node sinusoidal endothelial cell C-type lectin; PVR, poliovirus receptor; NSCLC, non-small cell lung cancer. 


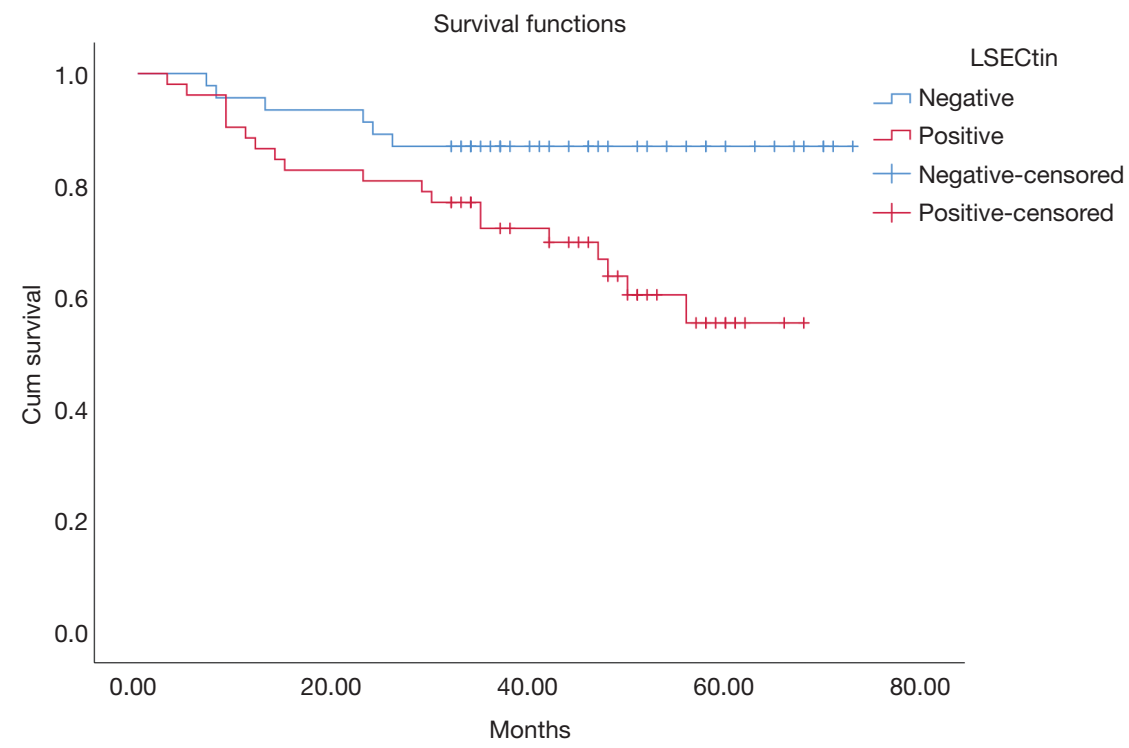

Figure 3 Kaplan-Meier survival analysis for OS in different LSECtin expression. LSECtin, liver and lymph node sinusoidal endothelial cell C-type lectin; OS, overall survival.
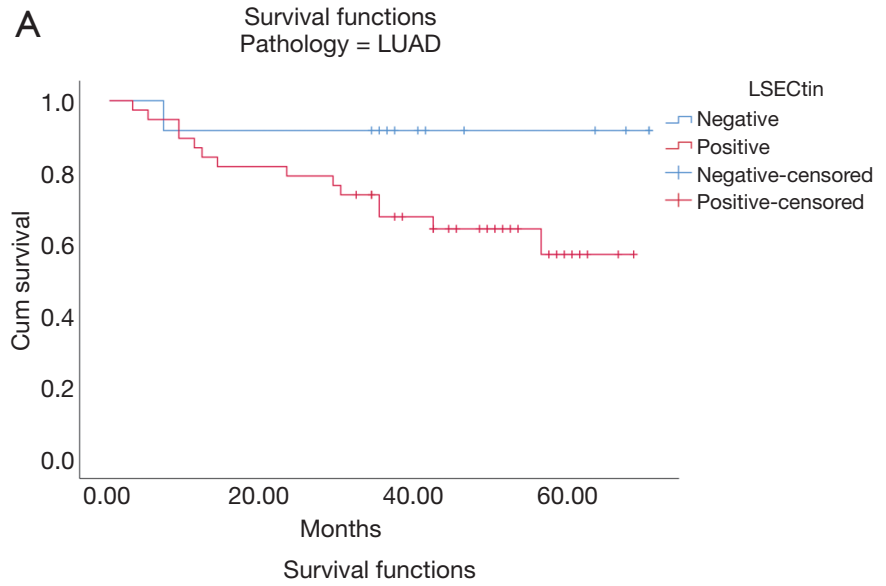

C

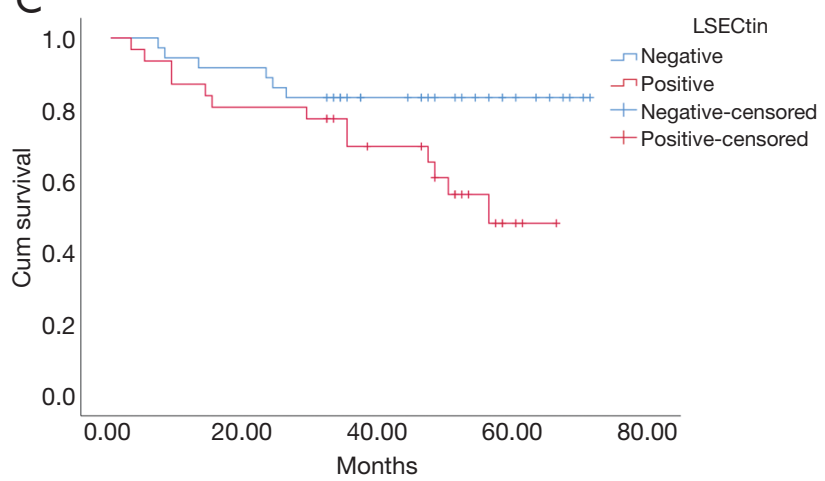

B Survival functions Pathology = LUSC

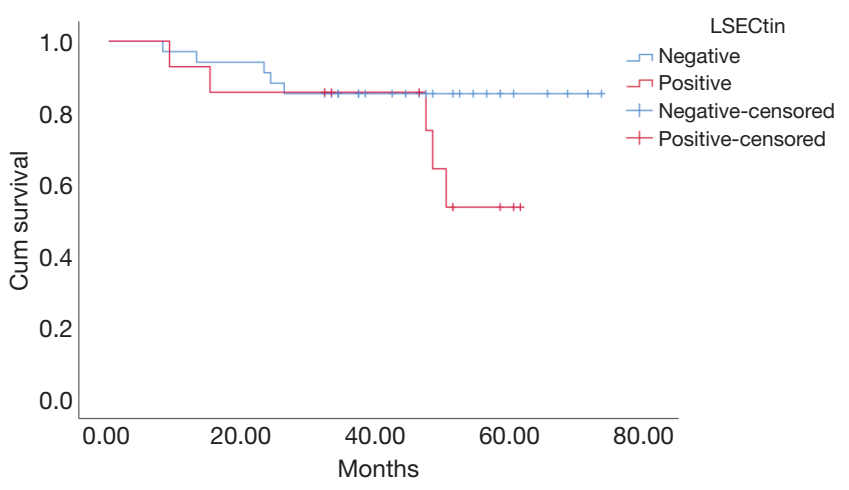

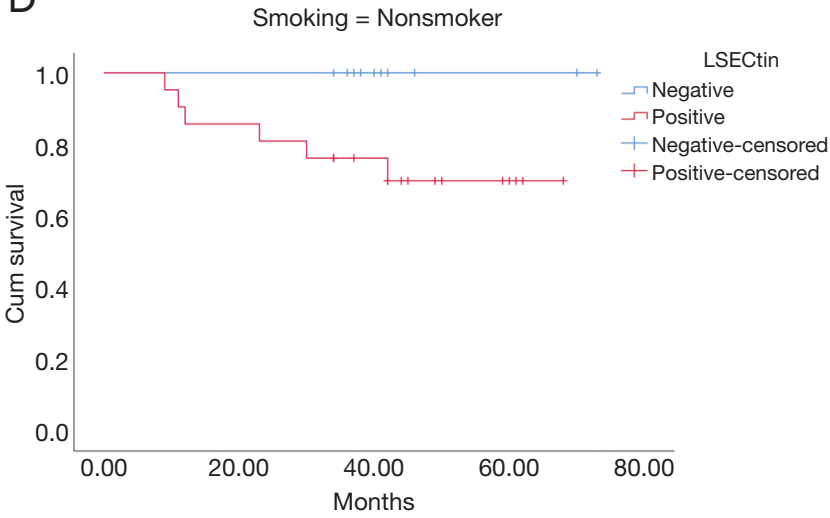

Figure 4 Kaplan-Meier survival analysis of OS in LSECtin expression with pathological pattern (A,B) and smoking history classification (C,D). LSECtin, liver and lymph node sinusoidal endothelial cell C-type lectin; LUAD, lung adenocarcinoma; LUSC, lung squamous cell carcinoma; OS, overall survival. 
Table 6 Univariate and multivariate analyses of overall survival in patients with NSCLC

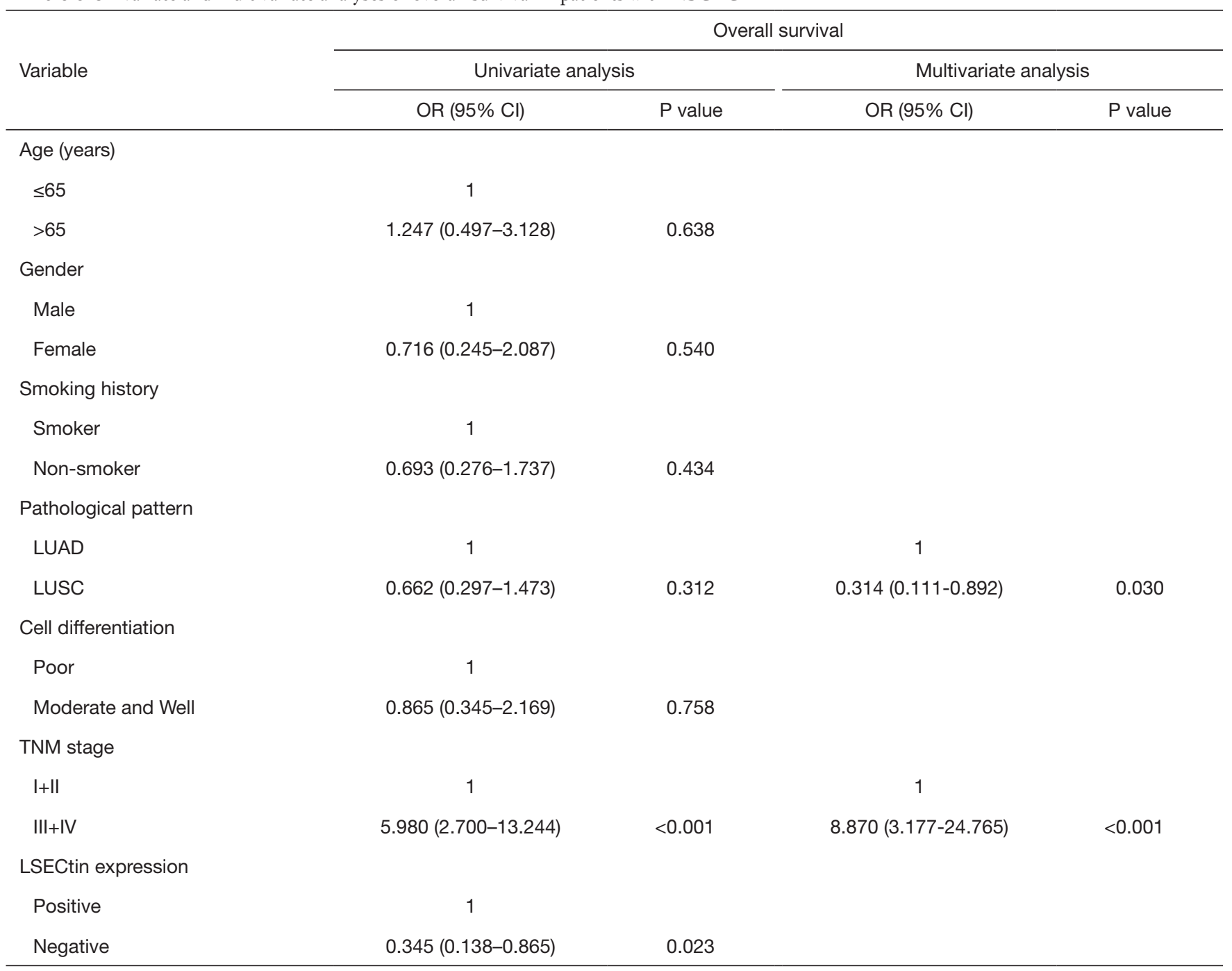

LSECtin, liver and lymph node sinusoidal endothelial cell C-type lectin; NSCLC, non-small cell lung cancer; LUAD, lung adenocarcinoma; LUSC, lung squamous cell carcinoma; TNM, tumor node metastasis; OR, odds ratio; Cl, confidence interval.

studies. The expression of highly inhibitory co-receptors, such as TIGIT and LAG-3, in lung NK cells has been correlated with mouse tumor lung metastasis (33). In immunotherapy resistance research, the exhausted $\mathrm{T}$ cells mediated by TIGIT and LAG-3 pathways were one of the important factors for the carboplatin chemoresistance in lung cancer (34). Therefore, future studies of ligands, including LSECtin and PVR, on different tumor cells will increase the selectivity and safety of the new-generation immune checkpoint blocking therapy. In the tumor microenvironment, the effect of each immune checkpoint pathway in tumor progression is not independent. The nonredundancy of multiple immunosuppressive pathways and the increasing accumulated clinical experience suggest that optimizing immunotherapy requires improvements by multiple aspects and pathways.

Smoking history has long been considered as a driving factor for lung cancer. Nonsmokers have accounted for 10$30 \%$ of all cases of lung cancer, and the cancer prevalence of nonsmokers exhibited an increasing trend in recent years $(35,36)$. The epidemiological study of lung cancer showed that nonsmokers had remarkable differences in clinical and molecular characteristics compared with smokers (37). Thus, this special group should be paid attention. In this study, LSECtin expression was more likely to be found in nonsmokers, and the LSECtin expression was more 
detrimental to OS in patients who never smoked than in smokers. Thus, subsequent studies focusing on LSECtin may help enhance the understanding of the mechanism of cancer progression in nonsmokers.

The results should be considered in the light of some limitations. First, this research was a regressive study based on a single center with small sample size, and the sample selection had unavoidable selection bias. Large sample sizes or multicenter studies should be required to validate current findings in the future. Second, this study did not classify oncogenic mutations in all cases. Many known targets, such as EGFR and ALK, have been related to prognosis and treatment options of patients with NSCLC. The joint analysis of LSECtin and these molecular markers will help further understand the effect of LSECtin on NSCLC. Finally, although the impact of LSECtin on the clinical prognosis and relationship between LSECtin and PVR in the protein level had been confirmed, studies on LSECtin are still very scarce in tumor research. The present results require further in vivo and in vitro experiments to explore its underlying mechanism.

In conclusion, LSECtin expression is an important potential indicator of prognosis in patients with NSCLC. In addition, a positive correlation was found between LSECtin and PVR, which are co-immunosuppressive ligands in NSCLC cells. Thus, a theoretical basis is provided to clinicians for follow-up strategy and future combination therapy of immune checkpoints.

\section{Acknowledgments}

Funding: This study was funded by the internal funding of Taihe Hospital (Project No. 2019JJXM007), the Science and Technology Development Fund, Macau SAR (File No. 0096/2018/A3,130/2017/A3, and 0099/2018/ A3) and the NSFC overseas and Hong Kong and Macao Scholars Cooperative Research Fund Project (Project No. 81828013).

\section{Footnote}

Reporting Checklist: The authors have completed the REMARK reporting checklist. Available at http://dx.doi. org/10.21037/atm-20-3665

Data Sharing Statement: Available at http://dx.doi. org/10.21037/atm-20-3665
Peer Review File: Available at http://dx.doi.org/10.21037/ atm-20-3665

Conflicts of Interest: All authors have completed the ICMJE uniform disclosure form (available at http://dx.doi. org/10.21037/atm-20-3665). The authors have no conflicts of interest to declare.

Ethical Statement: The authors are accountable for all aspects of the work in ensuring that the questions related to the accuracy or integrity of any part of the work have been appropriately investigated and resolved. The study was conducted in accordance with the Declaration of Helsinki (as revised in 2013) and had been approved by the Institutional Research Ethics Committee of Hubei Taihe Hospital (2020KS014). All patients signed informed consent.

Open Access Statement: This is an Open Access article distributed in accordance with the Creative Commons Attribution-NonCommercial-NoDerivs 4.0 International License (CC BY-NC-ND 4.0), which permits the noncommercial replication and distribution of the article with the strict proviso that no changes or edits are made and the original work is properly cited (including links to both the formal publication through the relevant DOI and the license). See: https://creativecommons.org/licenses/by-nc-nd/4.0/.

\section{References}

1. Hirsch FR, Scagliotti GV, Mulshine JL, et al. Lung cancer: current therapies and new targeted treatments. Lancet 2017;389:299-311.

2. Kishton RJ, Sukumar M, Restifo NP. Metabolic Regulation of T Cell Longevity and Function in Tumor Immunotherapy. Cell Metabolism 2017;26:94-109.

3. Topalian SL, Taube JM, Anders RA, et al. Mechanismdriven biomarkers to guide immune checkpoint blockade in cancer therapy. Nat Rev Cancer 2016;16:275-87.

4. Topalian SL, Hodi FS, Brahmer JR, et al. Safety, activity, and immune correlates of anti-PD-1 antibody in cancer. $\mathrm{N}$ Engl J Med 2012;366:2443-54.

5. Anderson AC, Joller N, Kuchroo VK. Lag-3, Tim-3, and TIGIT: co-inhibitory receptors with specialized functions in immune regulation. Immunity 2016;44:989-1004.

6. Zelba H, Bedke J, Hennenlotter J, et al. PD-1 and LAG-3 dominate checkpoint receptor-mediated $\mathrm{T}$ cell inhibition in renal cell carcinoma. Cancer Immunol Res 2019;7:1891-9. 
7. Burugu S, Gao D, Leung S, et al. LAG-3+ tumor infiltrating lymphocytes in breast cancer: clinical correlates and association with PD-1/PD-L1+ tumors. Ann Oncol 2017;28:2977-84.

8. He Y, Yu H, Rozeboom L, et al. LAG-3 Protein Expression in Non-Small Cell Lung Cancer and Its Relationship with PD-1/PD-L1 and Tumor-Infiltrating Lymphocytes. J Thorac Oncol 2017;12:814-23.

9. Waugh KA, Leach SM, Moore BL, et al. Molecular Profile of Tumor-Specific CD8+ T Cell Hypofunction in a Transplantable Murine Cancer Model. J Immunol 2016;197:1477-88.

10. Xu F, Liu J, Liu D, et al. LSECtin Expressed on Melanoma Cells Promotes Tumor Progression by Inhibiting Antitumor T-cell Responses. Cancer Res 2014;74:3418.

11. Liu W, Tang L, Zhang G, et al. Characterization of a novel C-type lectin-like gene, LSECtin demonstration of carbohydrate binding and expression in sinusoidal endothelial cells of liver and lymph node. J Biol Chem 2004;279:18748-58.

12. Zhao D, Han X, Zheng X, et al. The myeloid LSECtin is a DAP12-coupled receptor that is crucial for inflammatory response induced by Ebola virus glycoprotein. PLoS Pathog 2016;12:e1005542.

13. Gramberg T, Hofmann H, Möller P, et al. LSECtin interacts with filovirus glycoproteins and the spike protein of SARS coronavirus. Virology 2005;340:224-36.

14. Tang L, Yang J, Liu W, et al. Liver sinusoidal endothelial cell lectin, LSECtin, negatively regulates hepatic T-cell immune response. Gastroenterology 2009;137:1498-508. e1-5.

15. Zhao D, Zhang M, Wang M, et al. Up-regulation of Cbl-b is associated with LSECtin-mediated inhibition of different CD4+ T-cell subsets. Immunobiology 2013;218:602-8.

16. Liu D, Lu Q, Wang X, et al. LSECtin on tumorassociated macrophages enhances breast cancer stemness via interaction with its receptor BTN3A3. Cell Res 2019;29:365-78.

17. You H, Zhang Y, Lai H, et al. Prognostic significance of tumor poliovirus receptor and CTLA4 expression in patients with surgically resected non-small-cell lung cancer. J Cancer Res Clin Oncol 2020;146:1441-50.

18. Tang W, Pan X, Han D, et al. Clinical significance of CD8+ T cell immunoreceptor with Ig and ITIM domains+ in locally advanced gastric cancer treated with SOX regimen after D2 gastrectomy. Oncoimmunology 2019;8:e1593807.
19. Chauvin J-M, Pagliano O, Fourcade J, et al. TIGIT and PD-1 impair tumor antigen-specific CD8+ $T$ cells in melanoma patients. J Clin Invest 2015;125:2046-58.

20. Lee WJ, Lee YJ, Choi ME, et al. Expression of lymphocyte-activating gene 3 and T-cell immunoreceptor with immunoglobulin and ITIM domains in cutaneous melanoma and their correlation with programmed cell death 1 expression in tumor-infiltrating lymphocytes. J Am Acad Dermatol 2019;81:219-27.

21. Andrews LP, Marciscano AE, Drake CG, et al. LAG3 (CD223) as a cancer immunotherapy target. Immunol Rev 2017;276:80-96.

22. Tang L, Yang J, Tang X, et al. The DC-SIGN family member LSECtin is a novel ligand of CD44 on activated T cells. Eur J Immunol 2010;40:1185-91.

23. Liu X, Taftaf R, Kawaguchi M, et al. Homophilic CD44 interactions mediate tumor cell aggregation and polyclonal metastasis in patient-derived breast cancer models. Cancer Discov 2019;9:96-113.

24. Lai CJ, Lin CY, Liao WY, et al. CD44 promotes migration and invasion of docetaxel-resistant prostate cancer cells likely via induction of hippo-yap signaling. Cells 2019;8:295.

25. Zuo Y, Ren S, Wang M, et al. Novel roles of liver sinusoidal endothelial cell lectin in colon carcinoma cell adhesion, migration and in-vivo metastasis to the liver. Gut 2013;62:1169-78.

26. Na H, Liu X, Li X, et al. Novel roles of DC-SIGNR in colon cancer cell adhesion, migration, invasion, and liver metastasis. J Hematol Oncol 2017;10:28.

27. Hao X, Han F, Ma B, et al. SOX30 is a key regulator of desmosomal gene suppressing tumor growth and metastasis in lung adenocarcinoma. J Exp Clin Cancer Res 2018;37:111.

28. Yang Z, Li Q, Wang X, et al. C-type lectin receptor LSECtin-mediated apoptotic cell clearance by macrophages directs intestinal repair in experimental colitis. Proc Natl Acad Sci U S A 2018;115:11054-9.

29. O’Donnell JS, Madore J, Li XY, et al., editors. Tumor intrinsic and extrinsic immune functions of CD155. Semin Cancer Biol 2020;65:189-96.

30. Sanchez-Correa B, Valhondo I, Hassouneh F, et al. DNAM-1 and the TIGIT/PVRIG/TACTILE Axis: Novel Immune Checkpoints for Natural Killer Cell-Based Cancer Immunotherapy. Cancers 2019;11:877.

31. Dominguez-Soto A, Aragoneses-Fenoll L, Martin-Gayo E, et al. The DC-SIGN-related lectin LSECtin mediates 
antigen capture and pathogen binding by human myeloid cells. Blood 2007;109:5337-45.

32. Li XY, Das I, Lepletier A, et al. CD155 loss enhances tumor suppression via combined host and tumor-intrinsic mechanisms. J Clin Invest 2018;128:2613-25.

33. Ohs I, Ducimetière L, Marinho J, et al. Restoration of natural killer cell antimetastatic activity by IL12 and checkpoint blockade. Cancer Res 2017;77:7059-71.

34. Anestakis D, Petanidis S, Domvri K, et al. Carboplatin chemoresistance is associated with CD11b+/Ly6C+ myeloid release and upregulation of TIGIT and LAG3/

Cite this article as: Zhang Y, Lai H, Chen P, Li D, Khan I, Hsiao WLW, Fan X, Yao X, Wu Q, Wang M, Leung EL. Clinical significance of LSECtin and its association with PVR in non-small-cell lung cancer patients. Ann Transl Med 2020;8(21):1393. doi: 10.21037/atm-20-3665
CD160 exhausted T cells. Mol Immunol 2020;118:99-109.

35. Toh CK, Ong WS, Lim WT, et al. A decade of neversmokers among lung cancer patients-increasing trend and improved survival. Clin Lung Cancer 2018;19:e539-50.

36. Casal-Mourino A, Valdes L, Barros-Dios JM, et al. Lung cancer survival among never smokers. Cancer Lett 2019;451:142-9.

37. Sun S, Schiller JH, Gazdar AF. Lung cancer in never smokers-a different disease. Nat Rev Cancer 2007;7:778-90. 